\title{
UNIVERSITY OF BARCELONA RADIOCARBON DATES I
}

\section{J. S. MESTRES, GEMMA RAURET and J. F. GARCIA}

Radiocarbon Laboratory, University of Barcelona, Avenida Diagonal 647, 08028 Barcelona, Spain

\section{INTRODUCTION}

The Radiocarbon Laboratory at the University of Barcelona began its serial measurements in 1985. The following list contains dates obtained between 1985 and 1987. Both archaeological and geological samples were dated.

The measurement technique we use is liquid scintillation counting of benzene. A previous paper (Mestres, García \& Rauret 1991) describes sample pretreatment, synthesis and counting protocols and equipment.

Dates are expressed in radiocarbon years relative to A.D. 1950 based on 5568 years, the Libby half-life for ${ }^{14} \mathrm{C}$. Those dated since September 1987 are corrected for isotopic fractionation $\left(\delta^{13} \mathrm{C}\right.$ values are given relative to $\left.\mathrm{PDB}\right)$. The modern reference standard is the new NBS oxalic acid (factor 0.7459 ) corrected by isotopic fractionation. No corrections were made for ${ }^{14} \mathrm{C}$ reservoir effect. The radiocarbon dates are not dendrochronologically calibrated.

Errors quoted are one standard deviation and include the counting errors for the sample, the modern standard and background, and counting efficiency. More details on calculations are in Mestres, García and Rauret (1991). Descriptions, comments and references to publications are based on information supplied by collectors and submitters.

\section{ACKNOWLEDGMENTS}

We thank J. Merín, Dept. Analytical Chemistry, Univ. Barcelona, and C. Pachiaudi, Centre de Datations et d'Analyses Isotopiques, Univ. Lyon-I, for mass-spectrometric determination of $\delta^{13} \mathrm{C}$. We also wish to thank S. Soriano for her laboratory assistance.

\section{ARCHAEOLOGICAL SAMPLES}

Spain

\section{Cancho Roano series}

Samples from Cancho Roano, Zalamea de la Serena, Badajoz $\left(38^{\circ} 31^{\prime} \mathrm{N}, 5^{\circ} 40^{\prime} \mathrm{W}\right)$. Most samples are charcoal from different places of protohistoric funeral construction (6th, 5th century) (Maluquer 1983). Coll. and subm. by J. Maluquer, Dept. Prehistory and Archaeology, Univ. Barcelona.

UBAR-14. IAB-60

Charcoal from Sector 3, $2.37 \mathrm{~m}$ depth.

UBAR-22. IAB-54

$2520 \pm 120$

Charcoal from Sector 3, $2.37 \mathrm{~m}$ depth. 
UBAR-23. IAB-61

Charcoal from Sector 3, $2.37 \mathrm{~m}$ depth.

UBAR-29. IAB-55

$2470 \pm 100$

Charcoal from Sector 3.

UBAR-37. IAB-57

$2330 \pm 90$

Wheat seed carbonized from Sector 5, $1.34 \mathrm{~m}$ depth.

UBAR-19. IAB-68

$2720 \pm 100$

Charcoal from Room 5, 2 m depth.

UBAR-8. IAB-64

$2620 \pm 100$

Charcoal from Room 6, 2.20 m depth.

UBAR-17. IAB-66

$2450 \pm 60$

Charcoal from Room 8.

UBAR-27. IAB-65

$2360 \pm 100$

Charcoal from Room 8.

UBAR-18. IAB-63

$2710 \pm 100$

Charcoal from E 8.

UBAR-9. IAB-69

$2650 \pm 70$

Charcoal from eastern court on flagstone pavement, $3.70 \mathrm{~m}$ depth.

General Comment: dates agree with expected ages.

\section{Cerrillo Blanco - Porcuna series}

Samples of charcoal from Cerrillo Blanco, Porcuna, Córdoba $\left(37^{\circ} 52^{\prime} \mathrm{N}, 4^{\circ} 11^{\prime} \mathrm{W}\right)$. Samples are related to Greek pottery of 4 th century B.C. Coll. and subm. by J. Maluquer.

UBAR-24. IAB-58

UBAR-25. IAB-59

$2360 \pm 100$

General Comment: dates agree with expected ages.

\section{Pixarelles Cave series}

Charcoal from Pixarelles Cave, Tavertet $\left(42^{\circ} 1^{\prime} \mathrm{N}, 2^{\circ} 22^{\prime} \mathrm{E}\right)$. Samples are from different layers of two areas. Pixarelles Cave was used from Late Neolithic to Bronze Age (Rauret 1987). Series was dated to establish stratigraphic ${ }^{14} \mathrm{C}$ chronology and to relate it to cultural chronology (Rauret, Mestres \& García 1989). Coll. 1984 and subm. 1985 by A. M. Rauret, Inst. Archaeology, Univ Barcelona. 
UBAR-10. IAB-101/104/105/108

$2870 \pm 100$

Square A, Layer VIII.

UBAR-11. IAB-106

$3150 \pm 120$

Square A, Layer XIII.

UBAR-36. IAB-110

$2980 \pm 130$

Square B, Layer XIII.

UBAR-12. IAB-109

$3500 \pm 230$

Square A, Layer XVII.

UBAR-13. IAB-100/103

$3940 \pm 220$

Square W, Layer IV.

General Comment: dates agree with stratigraphic succession. Lack of resolution of dates from Late and Middle Bronze Age is settled by cultural affiliation.

\section{Bòbila Madurell series}

Charcoal from Bòbila Madurell, St. Quirze, Barcelona $\left(41^{\circ} 32^{\prime} \mathrm{N}, 2^{\circ} 05^{\prime} \mathrm{E}\right)$. Bóbila Madurell is extensive archaeologic site with approximately two different areas: 1) "Sepulcros de fosa" belongs to Middle Neolithic and Chalcolithic periods; 2) belongs to Early and Middle Bronze and Iron Age periods. In both sectors, there are some remains of Veraza Neolithic and some silo structures (Llongueras, Petit \& Marcet 1982; Llongueras, Marcet \& Petit 1986; Martín 1985; Martín et al. 1988). Three samples, UBAR-4, -5 and -6, were coll. before 1985 and subm. 1985 by M. Llongueras; the others were coll. and subm. 1987 by A. Martín, Servei Arqueol., Generalitat Catalunya.

UBAR-83. BM/B-4

$3620 \pm 80$

Sector B, Structure B-4, Level 6.

UBAR-84. BM/B-12

$5010 \pm 80$

Sector B, Pit-silo B-12.

UBAR-4. FOC-1

$3480 \pm 80$

Fire structure or possible silo in Middle Neolithic and Chalcolithic sector.

UBAR-5. FOC-2

$4940 \pm 250$

Same as above.

UBAR-6. HABITAT-1

$4970 \pm 80$

Structure, possible habitat or pit. 
UBAR-85. BM/D7b

Sector D, pit-silo or possible Oven D-7b, Level IV.

UBAR-86. BM/D-26

$2440 \pm 60$

Sector D, pit-silo D-26, Level 1.

UBAR-87. BM/D-38

$3350 \pm 90$

Sector D, pit-silo D-38, $50 \mathrm{~cm}$ depth.

UBAR-88. BM/D-40

$2700 \pm 120$

Sector D, pit D-40, 30-50 cm depth.

General Comment (AM): dates agree with materials that appeared in fill of silos. Only the date of UBAR-88, does not agree with expected age (2600-1900 B.C.); structure D-40 was the worst preserved.

\section{Can Tintoré series}

Charcoal from Can Tintoré, Gavà, Barcelona $\left(41^{\circ} 18^{\prime} \mathrm{N}, 2^{\circ} 0^{\prime} \mathrm{E}\right)$. This archaeologic site is underground mine (siliceous and phosphate-baricite) whose galleries were re-used as sepulchral site (Level IV). Occupation begins at Early Neolithic and ends at Late Neolithic with its main moment at Middle Neolithic (Villalba et al. 1986; Villalba, Blasco \& Edo 1989). UBAR-30, coll. before 1983; UBAR-46 to -49 coll. 1983; UBAR-41, 42 coll. 1986; subm. 1986 by J. Villalba, Mus. Gavà.

UBAR-30. (1) 7CT-28A-h-IV

$4710 \pm 130$

Mine 28, Gallery A, Level h, Layer IV. Previously dated as I-13099: $4820 \pm 100$ B.P.

UBAR-47. 2/7CT-28A-i-IV $4610 \pm 90$

Mine 28, Gallery A, Level i, Layer IV.

UBAR-49. 4/7 CT-28A-i-V $4740 \pm 90$

Mine 28, Gallery A, Level i, Layer V. No sepulchral level.

UBAR-46. 3/7 CT-28A-k-IV

$4380 \pm 80$

Mine 28, Gallery A, Level k, Layer IV.

UBAR-48. 5/8 CT-28C-1-I

$4690 \pm 100$

Mine 28, Gallery C, Level 1, Layer I.

UBAR-42. 86/CT-41-Ib-22

$4820 \pm 100$

Mine 41.

UBAR-41. 86/CT-49

$4970 \pm 110$

Mine 49. No sepulchral level. 
General comment (JV): dates confirm that sepulchral level was used continuously for short period of time corresponding to primary and successive burials. Date of UBAR-46 is younger than expected.

\section{Font de la Vena series}

Charcoal from Font de la Vena, Tavertet, Barcelona $\left(42^{\circ} 01^{\prime} \mathrm{N}, 2^{\circ} 26^{\prime} \mathrm{E}\right)$. Site is dolmen-tumulus with some Middle Neolithic materials (Montboló pottery). Coll. and subm. 1986 by J. Castells, Servei Arqueol., Generalitat Catalunya.

\section{UBAR-61. Font de la Vena-4}

Near dolmen at same depth as Montboló pottery, $63 \mathrm{~cm}$ depth.

\section{UBAR-60. Font de la Vena-3}

Near dolmen below Montboló pottery at $84 \mathrm{~cm}$ depth.

General comment: dates are older than expected by archaeologists, in both cases, 3500-3000 B.C.

\section{Cova-120 series}

Samples from Cova-120, Sales de Llierca, Girona $\left(42^{\circ} 17^{\prime} \mathrm{N}, 6^{\circ} 18^{\prime} \mathrm{E}\right)$. Cave was occupied in Middle Paleolithic, in Neolithic with silo structures, in Chalcolithic and in Bronze Age with burials, and later (Cent-Vint Group 1987). Previous dates are: Level I - UGRA-106: $440 \pm 160$ B.P.; UGRA-107: $3190 \pm 140$ B.P. (C. González, P. Sánchez \& M. Domingo 1985); Level II Gif-6925: $4240 \pm 70$ B.P. Coll. 1984 and subm. 1986 by G. Alcalde, Mus. Comarcal Garrotxa.

\section{UBAR-34. C-120-B-15-10}

$300 \pm 70$

Wood from Level I, previously dated as UGRA-106.

Comment (GA): date is much younger than expected, no archaeologic reasons are found.

\section{UBAR-31. C-120-F-16}

$8550 \pm 150$

Charcoal from $7 \mathrm{~m}^{2}$ in Level III.

Comment (GA): date is older than expected, probably due to contamination from mixing of samples in Paleolithic level.

UBAR-90. Aldovesta Carbó de Pi

$2360 \pm 60$

Charcoal from Aldovesta, Benifallet, Tarragona $\left(40^{\circ} 59^{\prime} \mathrm{N}, 0^{\circ} 31^{\prime} \mathrm{E}\right)$. Sample is related with Iron Age pottery with Phoenician amphora. Coll. 1986 and subm. 1987 by J. Sanmartí, Dept. Prehistory and Archaeology, Univ. Barcelona.

Comment: date agrees with expected age (4th century B.C.).

UBAR-89. CBA-1+2

$3960 \pm 60$

Charcoal from Brics d'Ardèvol, Pinós de Solsonés, Lleida $\left(42^{\circ} 6^{\prime} \mathrm{N}, 5^{\circ} 12^{\prime} \mathrm{E}\right)$. Site is Chalcolithic open-air settlement. Sample came from ten fire structures. Coll. and subm. 1987 by J Castany. 
Comment (JC): date agrees with age expected from Chalcolithic objects.

UBAR-57. Cova del Senglar

$4100 \pm 80$

Charcoal from Cova del Senglar, Albanyà, Girona $\left(42^{\circ} 9^{\prime} \mathrm{N}, 2^{\circ} 38^{\prime} \mathrm{E}\right)$. Site is cave with Late and Early Bronze and Early Neolithic occupations. Sample is from Neolithic level (sepulchral), 50-60 cm depth. Coll. and subm. 1986 by J. Tarrús and A. Bosch, Mus. Arqueol. Comarcal Banyoles.

Comment (JT): date is younger than expected (3900-3400 B.C.); pottery of this level belongs to Postcardial-Montboló complex of northeastern Catalonia.

\section{UBAR-101. Cova del Pasteral Z-III}

$\mathbf{5 2 7 0} \pm \mathbf{7 0}$

$\delta^{13} \mathrm{C}=-19.9 \%$

Bones from Cova del Pasteral, La Cellera de Ter, Girona $\left(41^{\circ} 58^{\prime} \mathrm{N}, 2^{\circ} 36^{\prime} \mathrm{E}\right)$. Site is Early Neolithic funeral cave (Bosch 1985). Sample comes from Zone-III. Coll. 1982 and subm. 1987 by A. Bosch and J. Tarrús.

Comment (JT): date is younger than expected (4200-3900 B.C.); cave pottery belongs to Epicardial complex of northeastern Catalonia.

UBAR-109. Cova de l'Avellaner Z-III

$5830 \pm 100$

$\delta^{13} \mathrm{C}=-20.2 \%$

Bones from Cova de l'Avellaner, Les Planes d'Hòstoles, Girona $\left(42^{\circ} 04^{\prime} \mathrm{N}, 2^{\circ} 32^{\prime} \mathrm{E}\right)$. Site is cave with three sepulchral areas (Bosch \& Tarrús 1987). Sample comes from Zone III. Coll. and subm. 1986 by A. Bosch and J. Tarrús.

Comment (JT): date agrees with expected age (3900-3600 B.C.). Pottery of this sepulchral cave belongs to Postcardial-Montboló complex of northeastern Catalonia.

\section{UBAR-100. Els Garrofers}

$5100 \pm 110$

Human bones from Els Garrofers del Torrent de Santa Maria, Vilanova i la Geltrú, Barcelona $\left(42^{\circ} 12^{\prime} \mathrm{N}, 1^{\circ} 41^{\prime} \mathrm{E}\right)$. Site is open-air sepulchral level of Middle Neolithic "sepulcros de fosa." Sample comes from 5-10 cm below present surface (Martín \& Miret, in press). Coll. 1985 and subm. 1987 by A. Martín.

Comment: date agrees with expected age.

UBAR-63. Cova dels Lladres-I

$5330 \pm 90$

Wood from Cova dels Lladres, Vacarisses, Barcelona $\left(41^{\circ} 36^{\prime} \mathrm{N}, 1^{\circ} 55^{\prime} \mathrm{E}\right)$. Site is Early Neolithic sepulchral cave. Sample comes from near Burial 1 (Ten 1989). Coll. before 1981 and subm. 1986 by R. Ten, Servei Arqueol., Generalitat Catalunya. Only acid pretreatment was given.

Comment: date agrees with expected age.

UBAR-72. Font Voltada-Foc 3

$10,920 \pm 240$

Charcoal from Cova de la Font Voltada, Montbrió de la Marca, Tarragona $\left(41^{\circ} 28^{\prime} \mathrm{N}, 1^{\circ} 18^{\prime} \mathrm{E}\right)$.

Site is small Late Epigravettian cave. Sample comes from $45-80 \mathrm{~cm}$ below datum. Coll. 1983/ 
85 and 1987 subm. by A. Mir, Mus. Arqueol. Salvador Vilaseca, Reus.

Comment: date agrees with expected age.

\section{GeOLOGICAL SAMPLES}

Spain

\section{Llobregós River terrace series}

Samples from Llobregŏs River, Torà, Lleida $\left(41^{\circ} 48^{\prime} \mathrm{N}, 1^{\circ} 25^{\prime} \mathrm{E}\right)$. Samples were taken from first terrace level $(+7 \mathrm{~m})$ above present alluvial plane of Llobregós River. These alluvial deposits are sandy and silty with many gypsum grains. Coll. 1987 by J. Escuer and subm. 1987 by J. Calvet, Dept. Dynamic Geol., Univ. Barcelona.

\section{UBAR-64. F-1'}

$4550 \pm 80$

Sample $2 \mathrm{~m}$ below top of terrace.

UBAR-65. F-1

$4800 \pm 140$

Sample $5 \mathrm{~m}$ below top.

General Comment (JC): dates are in Holocene period as expected.

\section{UBAR-96. Monistrol A-1}

$240 \pm 50$

$\delta^{13} \mathrm{C}=-26.6 \%$

Bituminous slime sediments from Monistrol d'Anoia, Barcelona $\left(41^{\circ} 27^{\prime} \mathrm{N}, 1^{\circ} 47^{\prime} \mathrm{E}\right)$. Sample coll. from upper level of Quaternary terrace $15 \mathrm{~m}$ above present level of Anoia River. Continental Upper Miocene deposits are below Quaternary sediments (Gallart 1981). Coll. and subm. 1987 by F. Gallart, Inst. J. Almera, CSIC, Barcelona.

Comment: date is much younger than expected, perhaps due to presence of bomb ${ }^{14} \mathrm{C}$ in sample.

UBAR-58. Mostra 4/Core E

$1510 \pm 80$

Slime sediments from Estany d'Ullastret, Pla. St. Andreu, Girona $\left(42^{\circ} 00^{\prime} \mathrm{N}, 3^{\circ} 05^{\prime} \mathrm{E}\right)$. Sample coll. when drilling Quaternary paleolake sediments, at 5.60-5.70 m depth from present surface. These sediments overlay Eocene materials (Santanach, Sanz de Galdeano \& Busquets 1980). Coll. 1984 and subm. 1986 by A. Esteban, Dept. Prehistory and Archaeology, Univ. Barcelona.

Comment: date agrees with age expected by submitter.

\section{Delta del Llobregat series}

Samples from delta of Llobregat River, Barcelona $\left(41^{\circ} 19^{\prime} \mathrm{N}, 2^{\circ} 07^{\prime} \mathrm{E}\right)$. From the present, continental surface sediment sequence in delta is: fluvial sediments, marine silty and clayed (Flandriense, Holocene) and fluvial sediments at base of Holocene and Pliocene deposits (Marqués 1984). Samples coll. 1985 by drilling and subm. 1986 by M. Manzano, Dept. Stratigraphy, Univ. Barcelona. 
UBAR-33. Delta Llobregat 7-34

Peat from marine clays (Flandriense).

UBAR-32. Delta Llobregat 7'-65

$>28,100$

Marine bivalve shell from pre-Holocene deposits.

General Comment (MM): as expected, using geochronologic criterion, base of Flandriense transgression is > 12,000 B.P.; top is $c a .2000$ B.P.

\section{La Marjal de Pego-Oliva series}

Peat from beach-marsh system in Pego-Oliva, La Safor, València. Samples obtained by drilling in different places within beach marsh (Pérez, Sanjaume \& Fumanal 1985; Fumanal, Sanjaume \& Pérez-Cueva 1985). Coll. 1986 and subm. 1987 by V. Rosselló, Dept. Geog., Univ. València.

UBAR-77. Pego 1/17

$10,120 \pm 460$

Drilling $1\left(38^{\circ} 53^{\prime} \mathrm{N}, 0^{\circ} 02^{\prime} \mathrm{W}\right),>20 \mathrm{~m}$ depth.

UBAR-78. Pego 3/16

$8300 \pm 170$

Drilling $3\left(38^{\circ} 53^{\prime} \mathrm{N}, 0^{\circ} 03^{\prime} \mathrm{W}\right), 16.5-15.8 \mathrm{~m}$ depth.

UBAR-43. Pego 5/19

$7120 \pm 90$

Drilling $5\left(38^{\circ} 51^{\prime} \mathrm{N}, 0^{\circ} 03^{\prime} \mathrm{W}\right)$, ca. $13 \mathrm{~m}$ depth.

UBAR-79. Pego 6/5

$2400 \pm 60$

Drilling $6\left(38^{\circ} 52^{\prime} \mathrm{N}, 0^{\circ} 03^{\prime} \mathrm{W}\right), 1.8-2.0 \mathrm{~m}$ depth.

UBAR-80. Pego 6/7

$3250 \pm 100$

Drilling $6\left(38^{\circ} 52^{\prime} \mathrm{N}, 0^{\circ} 03^{\prime} \mathrm{W}\right), 2.2-2.4 \mathrm{~m}$ depth.

UBAR-81. Pego 6/11

$3540 \pm 80$

Drilling $6\left(38^{\circ} 52^{\prime} \mathrm{N}, 0^{\circ} 03^{\prime} \mathrm{W}\right), 3.0-3.2 \mathrm{~m}$ depth.

UBAR-82. Pego 6/12

$5330 \pm 90$

Drilling $6\left(38^{\circ} 52^{\prime} \mathrm{N}, 0^{\circ} 03^{\prime} \mathrm{W}\right), 3.2-3.4 \mathrm{~m}$ depth.

UBAR-44. Pego 7/16

$7790 \pm 110$

Drilling $7\left(38^{\circ} 52^{\prime} \mathrm{N}, 0^{\circ} 04^{\prime} \mathrm{W}\right), c a .12 \mathrm{~m}$ depth.

UBAR-45. Pego 7/32

$>28,240$

Drilling $7\left(38^{\circ} 52^{\prime} \mathrm{N}, 0^{\circ} 04^{\prime} \mathrm{W}\right)$, ca. $19 \mathrm{~m}$ depth.

General Comment (VR): dates of beach-marsh system of Pego-Oliva are part of study of Quaternary littoral evolution of Mediterranean Sea Valencia coast. Dates show two general sea transgressions, one at Upper Pleistocene and the other at Holocene. 


\section{Catalonia coast series}

Samples from Catalonia continental shelf, Mediterranean Sea erosion. Samples obtained from outcropping of Quaternary units (R1 1989; R2 1986). Coll. 1984 M. Farrán and subm. 1987 by A. Maldonado, Inst. J. Almera, CSIC. Outer portion of shells was removed before dating (Mestres, García \& Rauret 1991).

UBAR-68. GC-84-6 TR-665

$2260 \pm 80$

Marine bivalve shell from Begur, Girona (41 $\left.54^{\prime} \mathrm{N}, 3^{\circ} 21^{\prime} \mathrm{E}\right), 20 \mathrm{~cm}$ depth.

UBAR-67. GC-84-6 TR-645

Marine bivalve shell from Palamós, Girona $\left(41^{\circ} 51^{\prime} \mathrm{N}, 3^{\circ} 14^{\prime} \mathrm{E}\right), 28 \mathrm{~cm}$ depth.

UBAR-69. GC-84-6 TR-619

$34,100 \pm 2200$

Marine bivalve shell from Palamós, Girona $\left(41^{\circ} 43^{\prime} \mathrm{N}, 3^{\circ} 6^{\prime} \mathrm{E}\right), 25 \mathrm{~cm}$ depth.

UBAR-70. GC-84-I TR-233

Marine shell from Tossa de Mar, Girona $\left(41^{\circ} 33^{\prime} \mathrm{N}, 2^{\circ} 47^{\prime} \mathrm{E}\right), 18 \mathrm{~cm}$ depth.

UBAR-71. TC-3 $(15 \mathrm{~cm})$

Marine shell from Besós River, Barcelona $\left(41^{\circ} 23^{\prime} \mathrm{N}, 2^{\circ} 16^{\prime} \mathrm{W}\right), 15 \mathrm{~cm}$ depth.

UBAR-66. CL-83-I K-94 depth.

Peat from L’Ampolla - Hospitalet de l'Infant, Tarragona $\left(40^{\circ} 54^{\prime} \mathrm{N}, 0^{\circ} 51^{\prime} \mathrm{W}\right), 75-80 \mathrm{~cm}$

General Comment (MF): results obtained for carbonates do not agree with expected ages. This must be due to recrystallization or transport of basal sediments by trawling. Results of sediment samples agree with expected ages.

\section{Balearic Islands series}

Samples from Balearic Islands continental shelf (water depth $<200 \mathrm{~m}$ ), obtained by drilling at different depths of Holocene level (Alonso et al. 1988). Coll. 1985 and subm. 1986 by B. Alonso and J. Guillem, Dept. Stratigraphy, Univ. Barcelona, except UBAR-26, coll. and subm. by I. Zamarreño, Inst. J. Almera, CSIC, Barcelona.

UBAR-54. 85-CA-56 (124)

$9910 \pm 140$

Carbonate rock accreted on algal core from Alcudia Bay, $\left(39^{\circ} 56^{\prime} \mathrm{N}, 3^{\circ} 15^{\prime} \mathrm{E}\right)$, at $124 \mathrm{~cm}$ depth from bottom of sea.

UBAR-55. 85-CA-52 (402-410)

$8570 \pm 110$

Carbonate rock accreted on algal core from Alcudia Bay $\left(39^{\circ} 53^{\prime} \mathrm{N}, 3^{\circ} 37^{\prime} \mathrm{E}\right)$, at $402-410 \mathrm{~cm}$ depth from bottom of sea. 
UBAR-53. 85-CA-51

$8670 \pm 90$

Carbonate rock accreted on algal core from Alcudia Bay $\left(39^{\circ} 51^{\prime} \mathrm{N}, 3^{\circ} 19^{\prime} \mathrm{E}\right)$, at $4 \mathrm{~cm}$ depth from bottom of sea.

UBAR-56. 85-CA-56 (29-37)

$13,100 \pm 200$

Carbonate rock accreted on algal core from Alcudia Bay $\left(39^{\circ} 56^{\prime} \mathrm{N}, 3^{\circ} 15^{\prime} \mathrm{E}\right)$, at $29-37 \mathrm{~cm}$ depth from bottom of sea.

UBAR-20. 85-CA-60 (160-190) A

$11,840 \pm 210$

Marine gastropod shell covered by contemporary organisms from south Majorca $\left(39^{\circ} 59^{\prime} \mathrm{N}\right.$, $3^{\circ} 35^{\prime} \mathrm{E}$ ), at $160-190 \mathrm{~cm}$ depth from bottom of sea.

UBAR-21. 85-CA-60 (160-190) B

$13,500 \pm 320$

Marine bivalve shells from south Majorca $\left(39^{\circ} 59^{\prime} \mathrm{N}, 3^{\circ} 35^{\prime} \mathrm{E}\right)$, at $160-190 \mathrm{~cm}$ depth from bottom of sea.

UBAR-52. 85-CA-9

$9610 \pm 100$

Recrystallized shell from south Majorca $\left(39^{\circ} 16^{\prime} \mathrm{N}, 2^{\circ} 51^{\prime} \mathrm{E}\right)$, at $125 \mathrm{~cm}$ depth from bottom of sea.

UBAR-62. 85-CA-47

MODERN

Marine bivalve shells from the channel between Majorca and Minorca $\left(39^{\circ} 54^{\prime} \mathrm{N}, 3^{\circ} 39^{\prime} \mathrm{E}\right)$, at $5 \mathrm{~cm}$ depth from bottom of sea.

UBAR-26. 35430

$810 \pm 60$

Coral from platform between Majorca and Ibiza $\left(38^{\circ} 40^{\prime} \mathrm{N}, 2^{\circ} 24^{\prime} \mathrm{E}\right)$. Dated by U/Th at 13,220 +450/-325 (J. Bischoff, pers. commun. 1987)

General Comment (JG): dates beginning of holocene transgression. Inversion between dates UBAR-56 and -54 could be attributed to nature of sample material, mixing and/or diagenesis.

\section{REFERENCES}

Alonso, B., Guillem, J., Canals, M., Serra, J., Acosta, J., Hernández, P., Sanz, J. L., Calafat, A. and Catafau, E. 1988 Los sedimentos de la plataforma continental balear. Acta Geol. Hispánica, 23(3): 185196.

Bosch, A. 1985 La cova del Pasteral, un jaciment neolític a la vall mitjana del Ter. Quaderns del CECB-1985, Banyoles: 29-56.

Bosch, A. and Tarrús, J. 1987 Enterramiento múltiple del Neolítico Antiguo Catalán. Cova de l'Avellaner (La Garrotxa, Gerona). Rev. Arqueol. 67: 20-24.

Cent-Vint Group 1987 Dinámica de la utilització de la Cova-120 per l'home en els darrers 6000 anys. In Eitizions del Centre d'Investigacions Arqueològiques de Girona. Sèr. Monogràfica 7.

Fumanal, P., Sanjaume, E. and Pérez-Cueva, A. 1985
Una playa Flandriense en el Marjal de Pego (Valencia). I Reuniao do Quaternario Iberico I: 407418.

Gallart, F. 1981 Neógeno superior y Cuaternario del Penedés (Catalunya, España). Acta Geol. Hispánica 16(3): 151-157.

González, C., Sánchez, P. and Domingo, M. 1985 University of Granada radiocarbon dates II. $R a$ diocarbon 27(3): 610-615.

Llongueras, M., Marcet, R. and Petit, M. 1986 Darrers treballs a la Bòbila Madurell (St. Quirze del Vallès, Vallès Occidental). Tribuna d'Arqueol. 198485. Edicions del Dept. Cultura, Generalitat de Catalunya, Barcelona: 25-34.

Llongueras, M., Petit, M. and Marcet, R. 1982 Bòbila Madurell, Sant Quirze del Vallès. Excavacions ar- 
queològiques a Catalunya als darrers anys. Edicions del Dept. Cultura, Generalitat de Catalunya, Barcelona: 85-57.

Maluquer, J. 1983 El santuari protohistòric de Zalamea la Serena. Tribuna d'Arqueol. 1982-83. Edicions Dept. Cultura, Generalitat de Catalunya, Barcelona: 31-38.

Martín, A. 1985 De la cultura de los "sepulcros de fosa" al grupo "Veraza" en el Vallés. Estudios de la Antigüedad 2, Prehistoria del Vallés. Edicions Univ. Autònoma de Barcelona.

Martín, A., Miret, J., Blanch, A., Aliaga, S., Enrich, R., Colomer, S., Albizuri, S. and Bosch, J. 1988 Campanya d'excavacions arqueològiques 1987-88 al jaciment de la Bòbila Madurell-Can Feu (Sant Quirze del Vallès, Vallès Occidental). Arraona Rev. Historia 3: 9-23.

Martín, A. and Miret, J., in press, Un enterrament neolític als "Garrofers del torrent de Sta. Maria" (Vilanova i la Geltrú, Garraf). Revisió de la cultura de sepulcres de fosa al Penedès. Gypsela 8.

Marqués, M. 1984 Les Formacions Quaternàries del Delta del Llobregat. Institut d'Estudis Catalans, Barcelona: $281 \mathrm{p}$.

Mestres, J. S., García, J. F. and Rauret, G. 1991 The Radiocarbon Laboratory at the University of Barcelona. Radiocarbon 33(1): 23-34.

Pérez, A., Sanjaume, E. and Fumanal, P. 1985 Marco y procesos continentales en la formación de la marisma de Pego. I Reuniao do Quaternario Iberico I: $435-445$.
Rauret, A. M. 1987 La seqüència estratigràfica de la Cova de les Pixarelles (Tavertet, Osona). Tribuna d'Arqueoi. 1986-87. Edicions Dept. Cultura, Generalitat de Catalunya, Barcelona: 59-68.

Rauret, A. M., Mestres, J. S. and García, J. F., in press, Relation between cultural and C-14 ages from a Bronze age site stratigraphy of Pixarelles cave, Catalonia, Spain. In Mook, W. G. and Waterbolk, H. T., eds., Archaeology and ${ }^{14}$ C. PACT 24.

R1 1989 Mapa Geológico de la Plataforma Continental Barcelona. IGME Servicio de Publicaciones del Ministerio de Industria: 35.

R2 1986 Mapa Geológico de la Plataforma Continental y Zonas Adyacentes Tarragona. IGME Servicio de Publicaciones del Ministerio de Industria: 42.

Santanach, P. F., Sanz de Galdeano, C. and Busquets, J. C. 1980 Neotectónica de las regiones Mediterráneas. Bol. Geol. Min. 91-92: 417-440.

Ten, R. $1989 \mathrm{El}$ jaciment arqueològic de la cova de les Animes (Matadepera) en el marc del Neolític Epicardial. I Trobada d'estudiosos de Sant Llorens del Munt i l'Obac, Matadepera 1987: 101-102.

Villalba, J., Bañolas, L., Arenas, J. and Alonso, M. 1986 Les mines neolítiques de Can Tintorer (Gavá). Excavaciones 1978-80. Excavacions arqueològiques a Catalunya 6. Edicions Dept. Cultura, Generalitat de Catalunya, Barcelona.

Villalba, J., Blasco, A. and Edo, M. 1989 La prehistòria al Baix Llobregat, estat de la qüestió. I Jornades Arqueològiques del Baix Llobregat, Castelldefels. Pre-actes, in press. 